\title{
COOLING OF A PLATE WITH GENERAL BOUNDARY CONDITIONS
}

\author{
F. D. ZAMAN and R. AL-KHAIRY
}

(Received 3 September 1997 and in revised form 21 October 1998)

\begin{abstract}
We consider steady state temperature distribution in a homogeneous rectangular infinite plate the lower part of which is cooled by a fluid flowing at a constant velocity while the upper part satisfies the general mixed boundary conditions. The Wiener-Hopf method has been used to obtain the solution in the infinite series form and some special cases have been discussed.
\end{abstract}

Keywords and phrases. Heat equation, cooling of plates, Wiener-Hopf method.

2000 Mathematics Subject Classification. Primary 35K20, 45E10; Secondary 80A20.

1. Introduction. The problem of heat conduction and cooling of plates and rods are important due to their industrial applications. The simplest problem is that of the one-dimensional heat conduction or linear heat flow. Carslaw and Jaeger [4] have discussed different aspects of linear heat flow in plates and rods having homogeneous or composite structure. In such problems, the boundary of the body under consideration is either assumed to be insulated or kept at a constant temperature. Some problems of practical interest however, require imposition of mixed boundary conditions. The classical transform or Fourier series techniques are then no longer applicable. An ingenious method of dealing with such problems is use of the Wiener-Hopf technique.

One of the early studies in this regard is by Caflisch and Keller [3] who have studied the problem of steady state heat conduction in a sufficiently hot plate being cooled by water flowing over its upper surface while its lower surface is insulated. The water adjacent to the hotter part is converted to steam, while the water adjacent to the cooler part is still in the liquid form. This situation results in the mixed boundary condition as the part which is covered by liquid satisfies the cooling condition while the remaining part may be treated as being insulated. The solution in terms of an infinite product involving the roots of a certain transcendental equation is then obtained using the Wiener-Hopf technique. More details about this technique may be found in the treatise by Noble [8].

Levine [7] also considered the same problem, but assumed a simpler representation of the sputtering temperature. In both cases, the authors first obtain an appropriate Green's function for the problem, thus reducing the problem to a singular integral equation which is then solved using the Wiener-Hopf technique.

Evans [5] considered the problem of lowering a long circular cylinder.

In this paper, we study a more general model of the steady state cooling problem by assuming general mixed boundary conditions over the upper surface of an infinite 
rectangular plate. We suppose that half of the upper surface is kept at a prescribed temperature while the other half is allowed a heat flux at a variable but prescribed rate. The lower surface of the plate is cooled by a fluid moving with a uniform speed. Some cases of practical interest can be obtained as a limiting case from this general situation.

2. Formulation of the problem. We consider an infinite rectangular plate of uniform thickness $h$, composed of uniform and homogeneous material possessing constant thermal diffusivity $k$ with the lower surface of the plate coinciding with $y=0$. We suppose that half of the upper surface $(y=h,-\infty<x<0)$ has been prescribed temperature $f(x)$, while the remaining half $(y=h, 0 \leq x<\infty)$ has heat flux given by $g(x)$. The lower surface of the plate is cooled by fluid moving with a uniform speed $v$ having a constant rate of cooling $\lambda$. Moreover temperature as $(x \rightarrow \pm \infty)$ is assumed to have a particular level of difference (see $[1,6])$. The physical considerations lead to the following mixed boundary value problem:

$$
\frac{\partial^{2} u}{\partial x^{2}}+\frac{\partial^{2} u}{\partial y^{2}}=2 s \frac{\partial u}{\partial x}, \quad 0 \leq y \leq h,-\infty<x<\infty,
$$

where $s=v / 2 k$.

(i) On the lower surface $y=0$,

$$
\frac{\partial u}{\partial y}+\lambda u=0, \quad \text { on } y=0,-\infty<x<\infty
$$

(ii) On the upper surface $y=h$,

$$
\begin{gathered}
u(x, y)=f(x), \quad \text { on } y=h, 0 \leq x<\infty, \\
\frac{\partial u}{\partial y}=g(x), \quad \text { on } y=h,-\infty<x<0 .
\end{gathered}
$$

(iii) In addition, we assume

$$
\begin{aligned}
u \rightarrow 1, & x \rightarrow \infty, \\
u \rightarrow 0, & x \rightarrow-\infty, \\
u & \sim O(1), \quad x \longrightarrow+0, \\
\frac{\partial u}{\partial y} & \sim O(1), \quad x \rightarrow-0 .
\end{aligned}
$$

The boundary data is assumed to be of exponential order, i.e.,

$$
\begin{array}{ll}
|f(x)|<c_{1} \exp \left(\tau_{-} x\right), & x \rightarrow \infty, \\
|g(x)|<c_{2} \exp \left(\tau_{+} x\right), & x \longrightarrow-\infty,
\end{array}
$$

where $c_{1}, c_{2}, \boldsymbol{\tau}_{-}$, and $\boldsymbol{T}_{+}$are constants and $-s<\boldsymbol{\tau}_{-}<\boldsymbol{\tau}_{+}<s$.

The above boundary value problem can be reduced to a more suitable form by setting

$$
u(x, y)=\phi(x, y) \exp (s x) .
$$


This yields,

$$
\frac{\partial^{2} \phi}{\partial x^{2}}+\frac{\partial^{2} \phi}{\partial y^{2}}-s^{2} \phi=0 .
$$

The boundary conditions now take the form

$$
\begin{gathered}
\frac{\partial \phi}{\partial y}+\lambda \phi=0, \quad \text { on } y=0,-\infty<x<\infty, \\
\phi(x, y)=f(x) \exp (-s x), \quad \text { on } y=h, 0 \leq x<\infty, \\
\frac{\partial \phi}{\partial y}=g(x) \exp (-s x), \quad \text { on } y=h,-\infty<x<0 .
\end{gathered}
$$

The behavior of the solution at $\pm \infty$ is now given by

$$
\begin{gathered}
\phi \sim \exp -s x, \quad \text { as } x \rightarrow \infty, \\
\phi \sim \epsilon \exp -s x \longrightarrow 0, \quad \text { as } x \longrightarrow-\infty,
\end{gathered}
$$

where $\epsilon$ is an arbitrary small real number. This condition can be derived from the behavior of the general solution of (2.8) as $x \rightarrow-\infty$ (see [2]).

The edge conditions at the point of separation of the two parts of the upper surface are

$$
\phi, \frac{\partial \phi}{\partial y} \sim O(1), \quad \text { as } x \rightarrow 0 \quad \text { on } y=h
$$

3. Reduction to the Wiener-Hopf equation. We define Fourier transform in $x$ and its inverse as

$$
\begin{gathered}
f^{*}(\alpha)=\int_{-\infty}^{\infty} f(x) \exp (i \alpha x) d x, \\
f(x)=\frac{1}{2 \pi} \int_{-\infty+i d}^{\infty+i d} f^{*}(\alpha) \exp (-i \alpha x) d \alpha,
\end{gathered}
$$

where $d$ is a constant chosen in the domain of analyticity of $f^{*}(\alpha)$ in the region $\tau_{-} \leq \operatorname{Im}(\alpha) \leq \tau_{+}$.

The half-range Fourier transforms are defined by

$$
\begin{aligned}
& f_{+}^{*}(\alpha)=\int_{0}^{\infty} f(x) \exp (i \alpha x) d x, \\
& f_{-}^{*}(\alpha)=\int_{-\infty}^{0} f(x) \exp (i \alpha x) d x,
\end{aligned}
$$

so that

$$
f^{*}(\alpha)=f_{+}^{*}(\alpha)+f_{-}^{*}(\alpha)
$$

It may be noted that if $f(x)=0\left(e^{\tau-x}\right)$ as $x \rightarrow \infty$ and $f(x)=0\left(e^{\tau+x}\right)$ as $x \rightarrow-\infty$, then $f_{+}^{*}(\alpha)$ is an analytic function of $\alpha$ in the upper half-plane $\operatorname{Im}(\alpha)>\tau_{-}$, while 
$f_{-}^{*}(\alpha)$ is thus in the lower half-plane $\operatorname{Im}(\alpha)<\tau_{+}$. From (2.9), $f^{*}(\alpha)$ is then analytic in $\tau_{-}<\operatorname{Im}(\alpha)<\tau_{+}$(Noble [8])

With these preliminaries, we apply the Fourier transform to the boundary value problem (2.8), (2.9), (2.10), and (2.11) to obtain

$$
\frac{d^{2} \phi^{*}(\alpha, y)}{d y^{2}}-\gamma^{2} \phi^{*}(\alpha, y)=0,
$$

where $\gamma(\alpha)=\sqrt{s^{2}+\alpha^{2}}$. The branch cuts of $\gamma$ are chosen such that $\gamma(0)=+s$.

$$
\begin{gathered}
\phi^{*^{\prime}}(\alpha, 0)+\lambda \phi^{*}(\alpha, 0)=0, \\
\phi_{+}^{*}(\alpha, h)=f_{+}^{*}(\alpha+i s), \quad \operatorname{Im}(\alpha)>\tau_{-}-s, \\
\phi_{-}^{*^{\prime}}(\alpha, h)=g_{-}^{*}(\alpha+i s), \quad \operatorname{Im}(\alpha)<\tau_{+}-s .
\end{gathered}
$$

The solution of the differential equation (3.5) that satisfies condition (3.6) is

$$
\phi^{*}(\alpha, y)=A(\alpha)(\gamma \cosh \gamma y-\lambda \sinh \gamma y) .
$$

Eliminating the unknown $A(\alpha)$ and using the decomposition (3.4) and the boundary conditions (3.7) and (3.8), we get, after some manipulation,

$$
\phi_{-}^{*}(\alpha, h)+f_{+}^{*}(\alpha+i s)=K(\alpha)\left(\phi_{+}^{*^{\prime}}(\alpha, h)+g_{-}^{*}(\alpha+i s)\right),
$$

where

$$
K(\alpha)=\frac{\cosh \gamma h-\lambda(\sinh \gamma h / \gamma)}{\gamma \sinh \gamma h-\lambda \cosh \gamma h}=\frac{G_{1}(\alpha)}{G_{2}(\alpha)}
$$

4. Solution of the Wiener-Hopf equation. Since $G_{1}(\alpha)$ and $G_{2}(\alpha)$ are entire functions of $\alpha$ having infinite number of zeros, $K(\alpha)$ being free of any branch points, we may use Weirstrass theorem to write $K(\alpha)=K_{+}(\alpha) K_{-}(\alpha)$, where

$$
K_{ \pm}(\alpha)=A^{1 / 2} \prod_{n=1}^{\infty}\left(\frac{\alpha \pm i \alpha_{n}}{\alpha \pm i \beta_{n}}\right),
$$

where

$$
A=\prod_{n=1}^{\infty}\left(\frac{\beta_{n}^{2}}{\alpha_{n}^{2}}\right)\left(\frac{\cosh s h-\lambda(\sinh s h / s)}{s \sinh s h-\lambda \cosh s h}\right),
$$

where $\pm i \alpha_{n}, \pm i \beta_{n}, n=1,2,3, \ldots$ are the simple zeros of $G_{1}(\alpha)$ and $G_{2}(\alpha)$, respectively. $K_{+}(\alpha)$ is free from zeros and poles in the upper half-plane and $K_{-}(\alpha)$ is free from zeros and poles in the lower half-plane.

Thus the Wiener-Hopf equation can be written as

$$
J(\alpha)=\frac{\phi_{-}^{*}(\alpha, h)}{K_{-}(\alpha)}-L_{-}(\alpha)=K_{+}(\alpha) \phi_{+}^{*^{\prime}}(\alpha)+L_{+}(\alpha),
$$

where

$$
L(\alpha)=L_{+}(\alpha)+L_{-}(\alpha)=K_{+}(\alpha) g_{-}^{*}(\alpha+i s)-\frac{f_{+}^{*}(\alpha+i s)}{K_{-}(\alpha)}
$$


The explicit expressions for $L_{ \pm}(\alpha)$ can be obtained by using the factorization theorem, Noble [8] as

$$
\begin{aligned}
& L_{+}(\alpha)=\frac{+1}{2 \pi i} \int_{-\infty+i c}^{\infty+i c}\left(K_{+}(\eta) g_{-}^{*}(\eta+i s)-\frac{f_{+}^{*}(\eta+i s)}{K_{-}(\eta)}\right) \frac{d \eta}{\eta-\alpha}, \\
& L_{-}(\alpha)=\frac{-1}{2 \pi i} \int_{-\infty+i c}^{\infty+i c}\left(K_{+}(\eta) g_{-}^{*}(\eta+i s)-\frac{f_{+}^{*}(\eta+i s)}{K_{-}(\eta)}\right) \frac{d \eta}{\eta-\alpha},
\end{aligned}
$$

We can argue that equation (4.3) defines an entire function since left-hand side is analytic in the lower have plane while right-hand side is analytic in the upper half plane, both sides being equal on the common strip of analyticity, are analytic continuation of each other. Using the asymptotic behavior of the functions and the infinite products, we can show that $J(\alpha)=0$. Hence

$$
\begin{gathered}
\phi_{-}^{*}(\alpha, h)=K_{-}(\alpha) L_{-}(\alpha), \\
\phi_{+}^{*^{\prime}}(\alpha)=-\frac{L_{+}(\alpha)}{K_{+}(\alpha)} .
\end{gathered}
$$

Using the factorization theorem of Noble [8], explicit forms for $L_{ \pm}(\alpha)$ can be obtained as

$$
\begin{aligned}
& L_{+}(\alpha)=-\frac{f_{+}^{*}(\alpha+i s)}{K_{-}(\alpha)}+\sum_{j=1}^{\infty} a_{j} \frac{g_{-}^{*}\left(-i \beta_{j}+i s\right)}{-i \beta_{j}-\alpha}+\sum_{j=1}^{\infty} a_{j}^{\prime} \frac{f_{+}^{*}\left(i \alpha_{j}+i s\right)}{i \alpha_{j}-\alpha}, \\
& L_{-}(\alpha)=K_{+}(\alpha) g_{-}^{*}(\alpha+i s)-\sum_{j=1}^{\infty} a_{j} \frac{g_{-}^{*}\left(-i \beta_{j}+i s\right)}{-i \beta_{j}-\alpha}-\sum_{j=1}^{\infty} a_{j}^{\prime} \frac{f_{+}^{*}\left(i \alpha_{j}+i s\right)}{i \alpha_{j}-\alpha},
\end{aligned}
$$

where

$$
\begin{aligned}
& a_{j}=A^{1 / 2} i\left(\beta_{j}-\alpha_{j}\right) \prod_{n=1, n \neq j}^{\infty}\left(\frac{\beta_{j}-\alpha_{n}}{\beta_{j}-\beta_{n}}\right), \\
& a_{j}^{\prime}=A^{-1 / 2} i\left(\alpha_{j}-\beta_{j}\right) \prod_{n=1, n \neq j}^{\infty}\left(\frac{\alpha_{j}-\beta_{n}}{\alpha_{j}-\alpha_{n}}\right) .
\end{aligned}
$$

5. Fourier transform inversion. The solution of the heat problem in the $(x, y)$ plane can be obtained by using the inversion formula (3.2) as

$\phi(x, y)=\frac{1}{2 \pi} \int_{-\infty+i d}^{\infty+i d}\left(\frac{\phi_{-}^{*}(\alpha, h)+f_{+}^{*}(\alpha+i s)}{\cosh \gamma h-\lambda(\sinh \gamma h / \gamma)}\right)\left(\cosh \gamma y-\lambda \frac{\sinh \gamma y}{\gamma}\right) \exp (-i \alpha x) d \alpha$,

where $d$ is chosen such that the contour of integration lie in the domain of convergence of the transform integral.

Noting that $\cosh \gamma y-\lambda(\sinh \gamma y / \gamma)$ has no branch points and so using its infinite product representation, we may write 


$$
\frac{\cosh \gamma y-\lambda(\sinh \gamma y / \gamma)}{\cosh \gamma h-\lambda(\sinh \gamma h / \gamma)}=D N(y) \prod_{n=1}^{\infty}\left(\frac{\alpha^{2}+\rho_{n}^{2}}{\alpha^{2}+\alpha_{n}^{2}}\right) \text {, }
$$

where

$$
\begin{gathered}
D=\prod_{n=1}^{\infty}\left(\frac{\alpha_{n}^{2}}{\rho_{n}^{2}}\right), \\
N(y)=\frac{\cosh s y-\lambda(\sinh s y / s)}{\cosh s h-\lambda(\sinh s h / s)},
\end{gathered}
$$

where $\pm i \rho_{n}$ are the simple zeros of $\cosh \gamma y-\lambda(\sinh \gamma y / \gamma)$. Thus

$$
\begin{aligned}
\phi(x, y) & =\frac{1}{2 \pi} D N(y) \int_{-\infty+i d}^{\infty+i d}\left(\phi_{-}^{*}(\alpha, h)+f_{+}^{*}(\alpha+i s)\right) \prod_{n=1}^{\infty}\left(\frac{\alpha^{2}+\rho_{n}^{2}}{\alpha^{2}+\alpha_{n}^{2}}\right) \exp (-i \alpha x) d \alpha \\
& =\frac{D}{2 \pi} N(y)\left(I_{1}(x)+I_{2}(x)\right),
\end{aligned}
$$

where

$$
\begin{aligned}
& I_{1}(x)=\int_{-\infty+i d}^{\infty+i d} f_{+}^{*}(\alpha+i s) \prod_{n=1}^{\infty}\left(\frac{\alpha^{2}+\rho_{n}^{2}}{\alpha^{2}+\alpha_{n}^{2}}\right) \exp (-i \alpha x) d \alpha, \\
& I_{2}(x)=\int_{-\infty+i d}^{\infty+i d} K_{-}(\alpha) H_{-}(\alpha) \prod_{n=1}^{\infty}\left(\frac{\alpha^{2}+\rho_{n}^{2}}{\alpha^{2}+\alpha_{n}^{2}}\right) \exp (-i \alpha x) d \alpha .
\end{aligned}
$$

If $x<0$, we close the contour of integration in the upper half-plane and for $x \geq 0$, the contour is chosen to be closed in the lower half-plane so that the above integrals are defined. This gives

$$
\begin{aligned}
& I_{3}(x)=\left\{\begin{array}{r}
2 \pi-\sum_{j=1}^{\infty} b_{j} f_{+}^{*}\left(-i \alpha_{j}+i s\right) \exp \left(-\alpha_{j} x\right) \\
\quad+\text { the contribution of the poles of } f_{+}^{*}(\alpha+i s) \text { if any }, \quad x \geq 0, \\
2 \pi-\sum_{j=1}^{\infty} b_{j} f_{+}^{*}\left(i \alpha_{j}+i s\right) \exp \left(\alpha_{j} x\right), \quad x<0,
\end{array}\right. \\
& I_{4}(x)=\left\{\begin{array}{c}
2 \pi-\sum_{j=1}^{\infty} b_{j} K_{-}\left(-i \alpha_{j}\right) L_{-}\left(-i \alpha_{j}\right) \exp \left(-\alpha_{j} x\right), \quad x \geq 0, \\
2 \pi-\sum_{j=1}^{\infty} b_{j} K_{-}\left(i \alpha_{j}\right) L_{-}\left(i \alpha_{j}\right) \\
\quad+\text { the contribution of the poles of } K_{-}(\alpha) H_{-}(\alpha), \quad x<0,
\end{array}\right.
\end{aligned}
$$

where

$$
b_{j}=\frac{\left(\alpha_{j}^{2}-\rho_{j}^{2}\right)}{2 \alpha_{j}} \prod_{n=1, n \neq j}^{\infty}\left(\frac{\alpha_{j}^{2}-\rho_{n}^{2}}{\left(\alpha_{j}+\alpha_{n}\right)\left(\alpha_{j}-\alpha_{n}\right)}\right) .
$$


6. Discussion and limiting cases. The temperature distribution in the plate can be obtained using (2.7) and (5.5). In a similar way the heat flux at the upper surface of the plate, which is of interest in many situations, can be obtained using expressions for $\phi_{+}^{*^{\prime}}(\alpha)$ obtainable from (4.7).

(a) Let us assume that the left half of the upper surface of the plate is insulated, i.e., $g(x)=0$, thus

$$
\phi(x, y)=\left\{\begin{aligned}
D N & (y) \sum_{j=1}^{\infty}\left[-b_{j} f_{+}^{*}\left(-i \alpha_{j}+i s\right)+\sum_{l=1}^{\infty} a_{l} c_{j} d_{j l} f_{+}^{*}\left(i \alpha_{l}+i s\right)\right] \exp \left(-\alpha_{j} x\right) \\
& + \text { the contribution of the poles of } f_{+}^{*}(\alpha+i s) \text { if any, } \quad x \geq 0, \\
D N & (y) \sum_{j=1}^{\infty}\left[-b_{j} f_{+}^{*}\left(i \alpha_{j}+i s\right)+\sum_{l=1}^{\infty} a_{l} c_{j}^{\prime} d_{j l}^{\prime} f_{+}^{*}\left(i \alpha_{l}+i s\right)\right] \exp \left(\alpha_{j} x\right) \\
& +\sum_{j=1}^{\infty} e_{j} f_{+}^{*}\left(i \beta_{j}+i s\right) \exp \left(\beta_{j} x\right), \quad x<0
\end{aligned}\right.
$$

where

$$
\begin{gathered}
c_{j}=b_{j} K_{-}(-i \alpha i), \quad c_{j}^{\prime}=b_{j} K_{-}\left(i \alpha_{i}\right)+\prod_{n=1, n \neq j}^{\infty} \frac{\alpha_{j}^{2}-\rho_{n}^{2}}{\left(\alpha_{l}+\beta_{n}\right)\left(\alpha_{l}-\alpha_{n}\right)}, \\
d_{j l}=\frac{1}{i\left(\alpha_{j}+\alpha_{l}\right)}, \quad d_{j l}^{\prime}=\frac{1}{i\left(\alpha_{l}-\alpha_{j}\right)}, \\
e_{j}=\prod_{n=1, n \neq j}^{\infty} \frac{\beta_{j}^{2}-\rho_{n}^{2}}{\left(\beta_{l}+\alpha_{n}\right)\left(\beta_{l}-\beta_{n}\right)} .
\end{gathered}
$$

(b) If the right half of the upper surface was heated uniformly from the point $x=0$ to $\infty$, then

$$
f(x)=a H(x),
$$

where $a$ is a constant and $H(x)$ is the Heaviside unit function. With this choice

$$
f_{+}^{*}(\alpha+i s)=\frac{a i}{\alpha+i s} .
$$

$f_{+}^{*}(\alpha+i s)$ has a simple pole at $\alpha=-i s$ in the lower half-plane, then for $s \neq \alpha_{n}, n=$ $1,2, \ldots$ we get

$$
\phi(x, y)=\left\{\begin{array}{c}
a D N(y) \sum_{j=1}^{\infty}\left[\frac{b_{i}}{\alpha_{j}-s}+\sum_{l=1}^{\infty} \frac{a_{l} c_{j} d_{j l}}{\alpha_{l}+s}\right] \exp \left(-\alpha_{j} x\right) \\
+\prod_{n=1}^{\infty} \frac{s^{2}-\rho_{n}^{2}}{s^{2}-\alpha_{n}^{2}} \exp (-s x), \quad x \geq 0 \\
a D N(y) \sum_{j=1}^{\infty}\left[\frac{-b_{j}}{\alpha_{j}+s}+\sum_{l=1}^{\infty} \frac{a_{l} c_{j}^{\prime} d_{j l}^{\prime}}{\alpha_{l}+s}\right] \exp \left(\alpha_{j} x\right) \\
+\sum_{j=1}^{\infty} \frac{e_{j}}{\beta_{j}+s} \exp \left(\beta_{j} x\right), \quad x<0 .
\end{array}\right.
$$


(c) If the right upper-half ( $y=h, 0 \leq x<\infty)$ was heated by an exponentially decaying behavior, i.e.,

$$
f(x)=b \exp (-a x)
$$

where $a>0$ and $b$ are constants.

Then

$$
f_{+}^{*}(\alpha+i s)=\frac{i b}{\alpha+i(s+a)} .
$$

Thus $f_{+}^{*}(\alpha+i s)$ has simple pole at $\alpha=-i(s+a)$ on the lower half-plane, then for $s+a \neq \alpha_{n}, n=1,2, \ldots$, we get

$$
\phi(x, y)=\left\{\begin{array}{c}
b D N(y) \sum_{j=1}^{\infty}\left[\frac{b_{i}}{\alpha_{j}-(s+a)}+\sum_{l=1}^{\infty} \frac{a_{l} c_{j} d_{j l}}{\alpha_{l}+(s+a)}\right] \exp \left(-\alpha_{j} x\right) \\
+\prod_{n=1}^{\infty} \frac{s^{2}-\rho_{n}^{2}}{s^{2}-\alpha_{n}^{2}} \exp (-s x), \quad x \geq 0 \\
b D N(y) \sum_{j=1}^{\infty}\left[\frac{-b_{j}}{\alpha_{j}+(s+a)}+\sum_{l=1}^{\infty} \frac{a_{l} c_{j}^{\prime} d_{j l}^{\prime}}{\alpha_{l}+(s+a)}\right] \exp \left(\alpha_{j} x\right) \\
+\sum_{j=1}^{\infty} \frac{e_{i}}{\beta_{j}+s} \exp \left(\beta_{j} x\right), \quad x<0 .
\end{array}\right.
$$

In a similar way, we may consider different forms of $f(x)$ and $g(x)$ to fit many physically interesting situations and obtain the solution in the infinite series form.

7. Numerical results. The numerical results of (6.5) and (6.8) for the temperature $u(0, y)$ at the midpoint $x=0$ are tabulated in Tables 7.1, 7.2, and 7.3. $u_{0}$ was calculated for suitable choices of the parameters $\Omega, s_{1}$, and $h_{1}$ where $\Omega=\lambda y, s_{1}=s y$, $h_{1}=y / h$. Table 7.1 shows that for fixed $s_{1}$ and $h_{1}$, the temperature $u_{0}$ of the uniformly heated plate with $a=1$, increase with increase of $\Omega$. Also for fixed $\Omega$ and $h_{1}, u_{0}$ decreases with increase of $s_{1}$. If we increase $\Omega$ more than 0.6 , the temperature $u_{0}$ begins to decrease until it reaches a constant value for example at $s_{1}=0.02$, $h_{1}=0.8$, we find that $u_{0}$ has nearly the same value $\left(u_{0}=0.398\right)$ for large values of $\Omega$ $(\Omega=300,400,500)$.

TABLE 7.1.

\begin{tabular}{l|c|c|c|c|c|c}
\hline \multicolumn{7}{c}{$s_{1}=0.001, h_{1}=0.8$} \\
\hline$\Omega$ & 0.00 & 0.04 & 0.08 & 0.2 & 0.4 & 0.6 \\
\hline$u_{0}(y)$ & 0.4998 & 0.5050 & 0.5109 & 0.5331 & 0.5996 & 0.7993 \\
\hline \multicolumn{7}{c}{$s_{1}=0.2, h_{1}=0.8$} \\
\hline$u_{0}(y)$ & 0.4577 & 0.4584 & 0.4628 & 0.4801 & 0.5218 & 0.6540 \\
\hline
\end{tabular}

Tables 7.2 and 7.3 shows that the temperature of the exponentially heated plate with $b=1$ depends on the exponent $a$. The temperature $u_{0}$ decreases with increase of $a$ which is clear from comparison of the two tables. 
TABLE 7.2.

\begin{tabular}{l|c|c|c|c|c|c}
\hline \multicolumn{7}{c}{$s_{1}=0.001, h_{1}=0.8, a=0.6$} \\
\hline$\Omega$ & 0.00 & 0.04 & 0.08 & 0.2 & 0.4 & 0.6 \\
\hline$u_{0}(y)$ & 0.3206 & 0.3225 & 0.3249 & 0.3347 & 0.3433 & 0.4087 \\
\hline \multicolumn{7}{c}{$s_{1}=0.02, h_{1}=0.8, a=0.6$} \\
\hline$u_{0}(y)$ & 0.3192 & 0.3210 & 0.3234 & 0.3332 & 0.3415 & 0.4063 \\
\hline
\end{tabular}

TABLE 7.3.

\begin{tabular}{l|c|c|c|c|c|c}
\hline \multicolumn{7}{c}{$s_{1}=0.001, h_{1}=0.8, a=1.0$} \\
\hline$\Omega$ & 0.00 & 0.04 & 0.08 & 0.2 & 0.4 & 0.6 \\
\hline$u_{0}(y)$ & 0.2638 & 0.2651 & 0.2670 & 0.2747 & 0.2771 & 0.3252 \\
\hline \multicolumn{7}{c}{$s_{1}=0.02, h_{1}=0.8, a=1.0$} \\
\hline$u_{0}(y)$ & 0.2629 & 0.2642 & 0.2660 & 0.2737 & 0.2760 & 0.3238 \\
\hline
\end{tabular}

\section{REFERENCES}

[1] R. K. Bera and A. Chakrabarti, Cooling of a composite slab in a two-fluid medium, Z. Angew. Math. Phys. 42 (1991), no. 6, 943-959. MR 93a:80002a. Zbl 739.73005.

[2] _ _ Cooling of an infinite slab in a two-fluid medium, J. Austral. Math. Soc. Ser. B 33 (1992), no. 4, 474-485. MR 93a:80002b. Zbl 755.73011.

[3] R. E. Caflisch and J. B. Keller, Quench front propagation, Nuclear Eng. and Design 65 (1981), 97-102.

[4] H. S. Carslaw and J. C. Jaeger, The Conduction of Heat in Solids, Clarendon Press, Oxford, 1959.

[5] D. V. Evans, A note on the cooling of a cylinder entering a fluid, IMA J. Appl. Math. 33 (1984), 49-54. Zbl 542.76115.

[6] H. G. Georgiadis, J. R. Barber, and F. Ben Ammar, An asymptotic solution for short-time transient heat conduction between two dissimilar bodies in contact, Quart. J. Mech. Appl. Math. 44 (1991), no. 2, 303-322. MR 92b:80005. Zbl 734.73005.

[7] Harold Levine, On a mixed boundary value problem of diffusion type, Appl. Sci. Res. 39 (1982), no. 4, 261-276. MR 84m:80010. Zbl 508.35076.

[8] B. Noble, Methods based on the Wiener-Hopf technique for the solution of partial differential equations, Pergamon Press, New York, 1958. MR 21\#1505. Zbl 082.32101.

Zaman: Department of Mathematical Sciences, King FAHD University of Petroleum AND Minerals, DHAHRAN 31261, SAUDi ARABIA

E-mail address: fzaman@kfupm. edu. sa

Al-Khairy: Department of Mathematics, Dammam College of Girls, Dammam, SAUdi ARABIA 


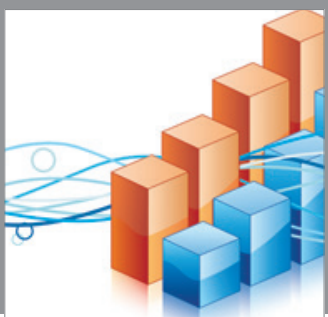

Advances in

Operations Research

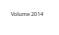

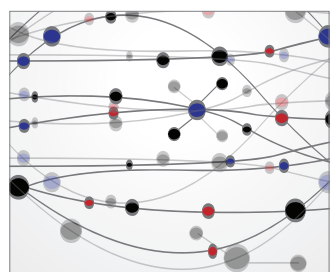

\section{The Scientific} World Journal
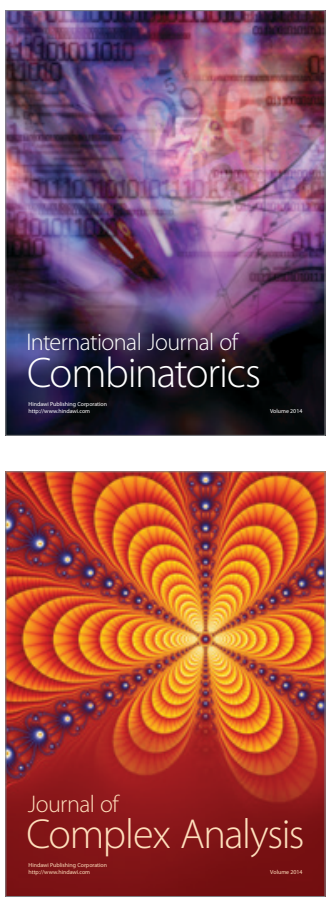

International Journal of

Mathematics and

Mathematical

Sciences
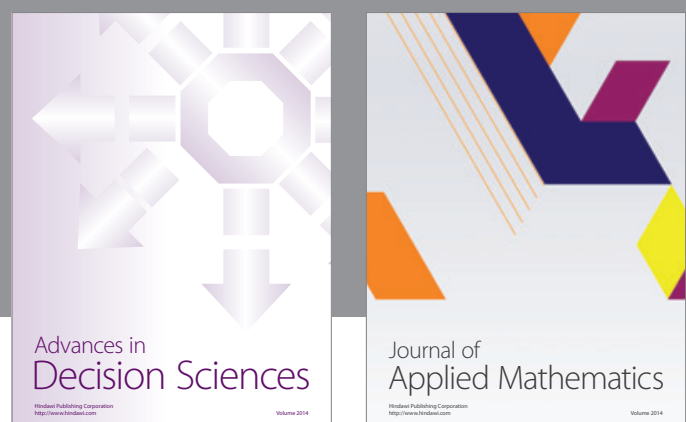

Journal of

Applied Mathematics
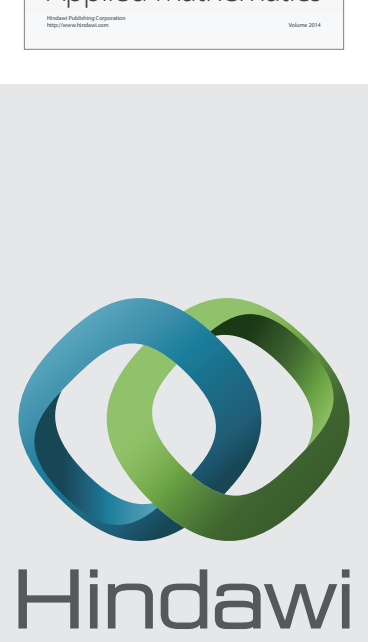

Submit your manuscripts at http://www.hindawi.com
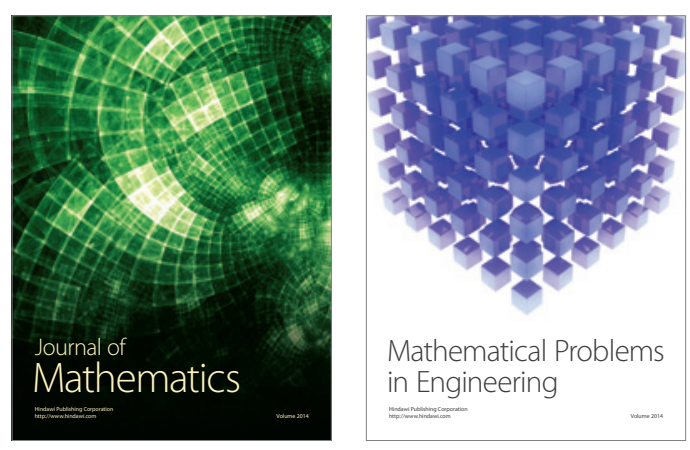

Mathematical Problems in Engineering
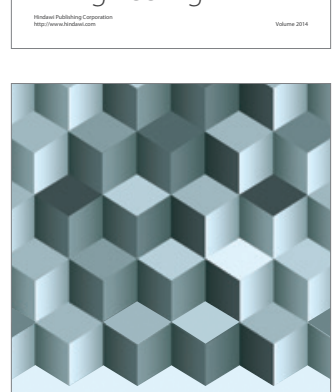

Journal of

Function Spaces
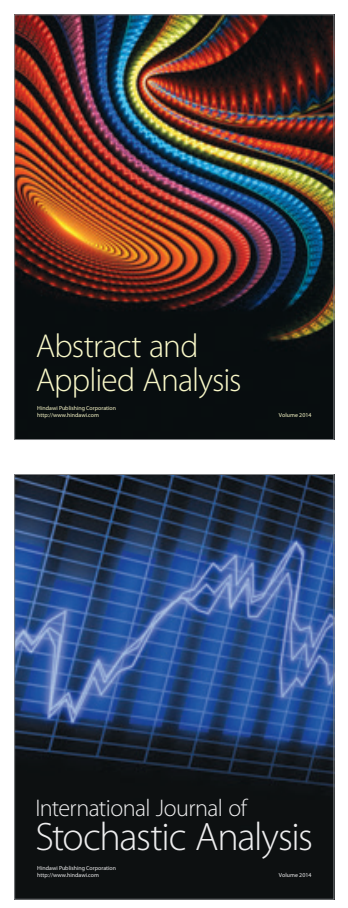

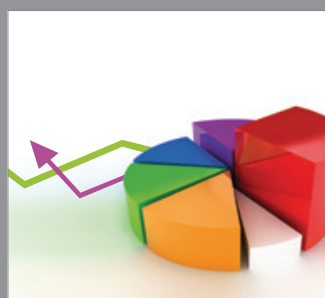

ournal of

Probability and Statistics

Promensencen
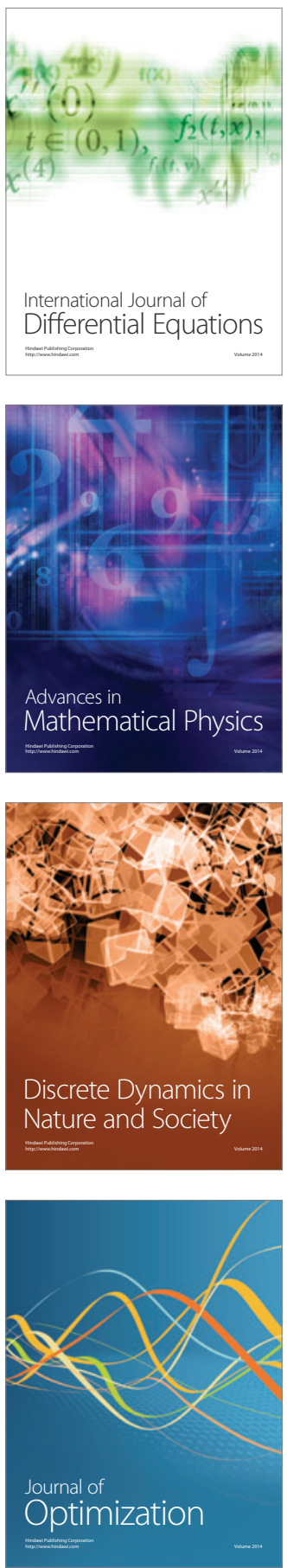